\title{
Discrete Entropic Uncertainty Relations Associated with FRFT $^{*}$
}

\author{
Guanlei $\mathrm{Xu}^{1}$, Xiaotong Wang ${ }^{2}$, Lijia Zhou ${ }^{1}$, Limin Shao ${ }^{1}$, Xiaogang $\mathrm{Xu}^{2}$ \\ ${ }^{1}$ Ocean department of Dalian Naval Academy, Dalian, China, 116018; ${ }^{2}$ Navgation department of Dalian Naval Academy, Dalian, \\ China, 116018. \\ Email: xgl_86@163.com
}

Received March, 2013.

\begin{abstract}
Based on the definition and properties of discrete fractional Fourier transform (DFRFT), we introduced the discrete Hausdorff-Young inequality. Furthermore, the discrete Shannon entropic uncertainty relation and discrete Rényi entropic uncertainty relation were explored. Also, the condition of equality via Lagrange optimization was developed, as shows that if the two conjugate variables have constant amplitudes that are the inverse of the square root of numbers of non-zero elements, then the uncertainty relations reach their lowest bounds. In addition, the resolution analysis via the uncertainty is discussed as well.
\end{abstract}

Keywords: Discrete Fractional Fourier Transform (DFRFT); Uncertainty Principle; Rényi Entropy; Shannon Entropy

\section{Introduction}

Uncertainty principle not only holds in analog signals, but also in discrete signals [1,2]. Recently, with the development of fractional Fourier transform (FRFT), ana$\log$ generalized uncertainty relations associated with FRFT have been carefully explored in some papers such as $[3,4,16]$, which effectively enrich the ensemble of FRFT. However, up till now there has been no reported article covering the discrete generalized uncertainty relations associated with discrete fractional Fourier transform (DFRFT). From the viewpoint of engineering application, discrete data are widely used and seem to be more profitable than the analog ones. Hence, there is enough need to explore discrete generalized uncertainty relations. DFRFT is the discrete version of FRFT $[5,6]$, which is applied in practical engineering fields. In this article we will discuss the entropic uncertainty relations $[7,8]$ associated with DFRFT. In this article, we made some contributions such as follows. The first contribution is that we extend the traditional Hausdorff-Young inequality to the FRFT domain with finite supports. It is shown that these bounds are connected with lengths of the supports and FRFT parameters. The second contribution is that we derived the Shannon entropic uncertainty principle in FRFT domain for discrete case, based on which we also derived the conditions when these uncertainty relations have the equalities via Lagrange optimi-

*This work was fully supported by the NSFC (61002052) and partly supported by the NSFC (61250006). zation. The third contribution is that we derived the Renyi entropic uncertainty principle in FRFT domain for discrete case. The final contribution is that we discussed the resolution in multiple FRFT domains as a succession of above derivative, including new proofs. In a word, there have been no reported papers covering these generalized discrete entropic uncertainty relations on FRFT.

\section{Preliminaries}

\subsection{FRFT and DFRFT}

First, confirm that you have the correct template for your paper size. This template has been tailored for output on the custom paper size $(21 \mathrm{~cm} * 28.5 \mathrm{~cm})$.

Before discussing the uncertainty principle, we introduce some relevant preliminaries. Here we first briefly review the definition of FRFT. For given analog signal $f(t) \in L^{1}(R) \cap L^{2}(R)$ and $\|f(t)\|_{2}=1$, its FRFT $[5,6]$ is defined as

$$
\begin{aligned}
& F_{\alpha}(u)=F_{\alpha}(f(t))=\int_{-\infty}^{\infty} f(t) K_{\alpha}(u, t) d t \\
& =\left\{\begin{array}{ll}
\sqrt{(1-i \cot \alpha) / 2 \pi} \cdot e^{\frac{i u^{2} \cot \alpha}{2}} \int_{-\infty}^{\infty} e^{\frac{-i \sin \alpha}{i t^{2} \cot \alpha}} f(t) d t & \alpha \neq n \pi \\
f(t) & \alpha=2 n \pi \\
f(-t) & \alpha=(2 n \pm 1) \pi
\end{array},\right.
\end{aligned}
$$

where $n \in \mathrm{Z}$ and $i$ is the complex unit, $\alpha$ is the transform parameter defined as that in $[5,6]$. In addition, 
$F_{\alpha} F_{\beta}(f(t))=F_{\alpha+\beta}(f(t))$. If $\alpha=-\beta, \quad F_{\alpha} F_{\beta}(f(t))=f(t)$, i.e., the inverse FRFT reads: $f(t)=\int_{-\infty}^{\infty} F_{\alpha}(u) K_{-\alpha}(u, t) d u$.

Let $X=\left\{x_{1}, x_{2}, x_{3}, \cdots, x_{N}\right\}=\{x(1), x(2), x(3), \cdots, x(N)\} \in C^{N}$ be a discrete time series with cardinality $N$ and $\|X\|_{2}=1$. Assume its DFRFT $\hat{X}_{\alpha}=\left\{\hat{x}_{1}, \hat{x}_{2}, \hat{x}_{3}, \cdots, \hat{x}_{N}\right\} \in C^{N}$ under the transform parameter $\alpha$.

Then the DFRFT [5] can be written as

$$
\begin{aligned}
& \hat{x}(k)=\sum_{l=1}^{N} \sqrt{(1-i \cot \alpha) / N} \cdot e^{\frac{i k^{2} \cot \alpha}{2}} e^{\frac{-i k n}{N \sin \alpha}} e^{\frac{i i^{2} \cot \alpha}{2 N^{2}}} x(n) \\
& =\sum_{l=1}^{N} u_{\alpha}(k, n) \cdot x(n), \quad 1 \leq n, k \leq N .
\end{aligned}
$$

Also, we can rewrite the definition (2) as

$$
\hat{X}_{\alpha}=U_{\alpha} X \text {, }
$$

where $U_{\alpha}=\left[u_{\alpha}(k, n)\right]_{N \times N}$.

Clearly, for DFRFT we have the following properties[5]:

$$
\begin{gathered}
U_{\beta}\left(U_{\alpha} X\right)=U_{\alpha}\left(U_{\beta} X\right)=U_{\alpha+\beta} X=U_{2 k \pi+\alpha+\beta} X, \\
U_{-\alpha}\left(U_{\alpha} X\right)=X=U_{2 k \pi} X, \\
\left\|\hat{X}_{\alpha}\right\|_{2}=\left\|U_{\alpha} X\right\|_{2}=1 .
\end{gathered}
$$

In the follows, we will assume that the transform parameter $0 \leq \alpha<2 \pi$ and $\alpha \neq \pi$. The max difference between the discrete and analog definitions is the support: one is finite and discrete and the other one is infinite and analog.

\subsection{Shannon Entropy and Rényi Entropy}

For any discrete random variable $x_{n}(n=1, \cdots, N)$ and its probability density function $p\left(x_{n}\right)$, the Shannon entropy [9] and the Rényi Entropy [10] is defined as, respectively

$$
\begin{gathered}
H\left(x_{n}\right)=\sum_{n=1}^{N}\left|p\left(x_{n}\right)\right| \ln \left|p\left(x_{n}\right)\right|, \\
H_{\vartheta}\left(x_{n}\right)=\frac{1}{1-\vartheta} \cdot \ln \left(\sum_{n=1}^{N}\left|p\left(x_{n}\right)\right|^{\vartheta}\right) .
\end{gathered}
$$

Hence, in this article, we know that for any DFRFT $\hat{X}_{\alpha}=\left\{\hat{x}_{1}, \hat{x}_{2}, \hat{x}_{3}, \cdots, \hat{x}_{N}\right\} \in C^{N}$, the Shannon entropy and the Rényi Entropy associated with DFRFT is defined as, respectively

$$
\begin{gathered}
H\left(\hat{x}_{\alpha}\right)=\sum_{n=1}^{N}\left|\hat{x}_{\alpha}(n)\right|^{2} \ln \left|\hat{x}_{\alpha}(n)\right|^{2}, \\
H_{\vartheta}\left(\hat{x}_{\alpha}\right)=\frac{1}{1-\vartheta} \cdot \ln \left(\sum_{n=1}^{N}\left|\hat{x}_{\alpha}(n)\right|^{2 \vartheta}\right) .
\end{gathered}
$$

Clearly, if $\vartheta \rightarrow 1, H_{\vartheta}\left(\hat{x}_{\alpha}\right) \rightarrow H\left(\hat{x}_{\alpha}\right)$.

\subsection{Discrete Hausdorff-Young Inequality Associated with DFRFT}

Let $\quad X=\left\{x_{1}, x_{2}, x_{3}, \cdots, x_{N}\right\}=\{x(1), x(2), x(3), \cdots, x(N)\} \in C^{N}$ be a discrete time series with cardinality $N$ and its DFRFT $\hat{X}_{\gamma}=\left\{\hat{X}_{1}, \hat{X}_{2}, \hat{X}_{3}, \cdots, \hat{X}_{N}\right\} \in C^{N}$ with $\hat{X}_{\gamma}=U_{\gamma} X$ and the transform parameter $\gamma$.

Since $\|X\|_{2}=1,\left\|\hat{X}_{\beta}\right\|_{2}=\left\|U_{\gamma} X\right\|_{2}=1$ from Parseval's theorem. Here $\|X\|_{2}=\left[\sum_{n=1}^{N}\left|X_{n}\right|^{2}\right]^{\frac{1}{2}}$. Clearly, we can obtain $\left\|U_{\gamma} X\right\|_{\infty} \leq M_{\gamma}\|X\|_{1}$ with $M=\left\|U_{\gamma}\right\|_{\infty}$.

Here $\left\|U_{\gamma}\right\|_{\infty}=\sup \left|u_{r}(l)\right|$ with $U_{\gamma}=\left\{u_{r}(l)\right\}, l=1, \cdots, N$.

Hence, we have $\left\|U_{\gamma} X\right\|_{\infty} \leq M_{\gamma}\|X\|_{1}$ with $M_{\gamma}=\left\|U_{\gamma}\right\|_{\infty}$.

Then from Riesz's theorem [11,12], we can obtain the discrete Hausdorff-Young inequality

$\left\|U_{\gamma} X\right\|_{q} \leq\left(M_{\gamma}\right)^{\frac{2-p}{p}}\|X\|_{p}$ with $1<p \leq 2$ and $\frac{1}{p}+\frac{1}{q}=1$.

Set $U_{\gamma}=U_{\alpha-\beta}$, then $U_{\gamma}=U_{\alpha-\beta}=U_{\alpha} U_{-\beta}$, we obtain

$\left\|U_{\alpha} U_{-\beta} X\right\|_{q} \leq\left(M_{\gamma}\right)^{2-p}\|X\|_{p}$ with $M_{\gamma}=\left\|U_{\alpha-\beta}\right\|_{\infty}=\left\|U_{\alpha} U_{-\beta}\right\|_{\infty}$.

Let $Y=U_{-\beta} X$, then $X=U_{\beta} Y$. In addition, from the property of DFRFT we can have

$$
\begin{gathered}
M_{\alpha-\beta}=\left\|U_{\alpha} U_{-\beta}\right\|_{\infty}=\sqrt{\frac{1}{N \cdot|\sin (\alpha-\beta)|}} \text {. Hence we obtain } \\
\left\|U_{\alpha} Y\right\|_{q} \leq\left(M_{\alpha-\beta}\right)^{\frac{2-p}{p}}\left\|U_{\beta} Y\right\|_{p} \text { with } M_{\alpha-\beta}=\sqrt{\frac{1}{N \cdot \sin (\alpha-\beta) \mid}} .
\end{gathered}
$$

Since the value of $X$ can be taken arbitrarily in $C^{N}$, $Y$ can also be taken arbitrarily in $C^{N}$. Therefore, we can obtain the following lemma.

Lemma 1: For any given discrete time series

$$
X=\left\{x_{1}, x_{2}, x_{3}, \cdots, x_{N}\right\}=\{x(1), x(2), x(3), \cdots, x(N)\} \in C^{N}
$$

with cardinality $N$ and $\|X\|_{2}=1, U_{\alpha}\left(U_{\beta}\right)$ is the DFRFT transform matrix associated with the transform parameter $\alpha$ ( $\beta$, respectively), then we can obtain the generalized discrete Hausdorff-Young inequality

$$
\left\|U_{\alpha} X\right\|_{q} \leq(N \cdot|\sin (\alpha-\beta)|)^{\frac{p-2}{2 p}}\left\|U_{\beta} X\right\|_{p}
$$

with $1<p \leq 2$ and $\frac{1}{p}+\frac{1}{q}=1$.

Clearly, this is the discrete version of HausdorffYoung inequality. In the next sections, we will use this lemma to prove the new uncertainty relations.

\section{The Uncertainty Relations}

\subsection{Shannon Entropic Principle}

Theorem 1: For any given discrete time series $X=\left\{x_{1}, x_{2}, x_{3}, \cdots, x_{N}\right\}=\{x(1), x(2), x(3), \cdots, x(N)\} \in \quad C^{N}$ with cardinality $N$ and $\|X\|_{2}=1, \hat{x}_{\alpha}\left(\hat{x}_{\beta}\right)$ is the DFRFT series associated with the transform parameter $\alpha(\beta$, respectively), $N_{\alpha}\left(N_{\beta}\right)$ counts the non-zero elements of $\hat{x}_{\alpha}\left(\hat{x}_{\beta}\right.$, respectively), then we can obtain the generalized discrete Shannon entropic uncertainty relation

$$
H\left(\hat{x}_{\alpha}\right)+H\left(\hat{x}_{\beta}\right) \geq \ln (|N \cdot \sin (\alpha-\beta)|),
$$


where

$$
\begin{gathered}
H\left(\hat{x}_{\alpha}\right)=-\sum_{n=1}^{N}\left[\left(\ln \left|\hat{x}_{\alpha}(n)\right|^{2}\right) \cdot\left|\hat{x}_{\alpha}(n)\right|^{2}\right], \\
H\left(\hat{x}_{\beta}\right)=-\sum_{m=1}^{N}\left[\left(\ln \left|\hat{x}_{\beta}(m)\right|^{2}\right) \cdot\left|\hat{x}_{\beta}(m)\right|^{2}\right],
\end{gathered}
$$

which are Shannon entropies. The equality in (3) holds iff $\left|\hat{x}_{\alpha}\right| \equiv \frac{1}{\sqrt{N_{\alpha}}}$ and $\left|\hat{x}_{\beta}\right| \equiv \frac{1}{\sqrt{N_{\beta}}}$.

Proof: From lemma 1, we have

$$
\frac{\left(\left.N \cdot|\sin (\alpha-\beta)|\right|^{\frac{p-2}{2 p}}\left(\sum_{m=1}^{N}\left|\hat{x}_{\beta}(m)\right|^{p}\right)^{\frac{1}{p}}\right.}{\left(\sum_{n=1}^{N}\left|\hat{x}_{\alpha}(n)\right|^{\frac{p}{p-1}}\right)^{\frac{p-1}{p}}} \geq 1 .
$$

Take natural logarithm in both sides in above inequality, we can obtain

$$
T(p) \geq 0,
$$

where

$$
\begin{gathered}
T(p)=\frac{p-2}{2 p} \ln (N \cdot|\sin (\alpha-\beta)|)+\frac{1}{p} \ln \left(\sum_{m=1}^{N}\left|\hat{x}_{\beta}(m)\right|^{p}\right) \\
-\frac{p-1}{p} \ln \left(\sum_{n=1}^{N}\left|\hat{x}_{\alpha}(n)\right|^{\frac{p}{p-1}}\right) .
\end{gathered}
$$

Since $1<p \leq 2$ and $\|X\|_{2}=1$, we know $T(2)=0$. Note $T(p) \geq 0$ if $1<p \leq 2$. Hence, $T^{\prime}(p) \leq 0$ if $p=2$. Since

$$
\begin{aligned}
T^{\prime}(p) & =\frac{1}{p^{2}} \ln (N \cdot|\sin (\alpha-\beta)|)-\frac{1}{p^{2}} \ln \left(\sum_{m=1}^{N}\left|\hat{x}_{\beta}(m)\right|^{p}\right) \\
& +\frac{1}{p} \frac{\sum_{m=1}^{N}\left[\left(\ln \left|\hat{x}_{\beta}(m)\right|\right) \cdot\left|\hat{x}_{\beta}(m)\right|^{p}\right]}{\sum_{m=1}^{N}\left|\hat{x}_{\beta}(m)\right|^{p}} \\
& -\frac{1}{p^{2}} \ln \left(\sum_{n=1}^{N}\left|\hat{x}_{\alpha}(n)\right|^{\frac{p}{p-1}}\right) \\
& +\frac{1}{p(p-1)} \frac{\sum_{n=1}^{N}\left[\left|\hat{x}_{\alpha}(n)\right| \frac{p}{p-1} \cdot \ln \left(\left|\hat{x}_{\alpha}(n)\right|\right)\right]}{\sum_{n=1}^{N}\left|\hat{x}_{\alpha}(n)\right|^{\frac{p}{p-1}}},
\end{aligned}
$$

we can obtain the final result in theorem 1 .

Now consider when the equality holds. From theorem 1 , that the equality holds in (3) implies that $H\left(\hat{x}_{\alpha}\right)+H\left(\hat{x}_{\beta}\right)$ reaches its minimum bound, which means that i.e.

Minimize $H\left(\hat{x}_{\alpha}\right)+H\left(\hat{x}_{\beta}\right)$ subject to $\left\|\hat{x}_{\alpha}\right\|_{2}=\left\|\hat{x}_{\beta}\right\|_{2}=1$,

Minimize

$-\sum_{n=1}^{N}\left[\left(\ln \left|\hat{x}_{\alpha}(n)\right|^{2}\right) \cdot\left|\hat{x}_{\alpha}(n)\right|^{2}\right]-\sum_{m=1}^{N}\left[\left(\ln \left|\hat{x}_{\beta}(m)\right|^{2}\right) \cdot\left|\hat{x}_{\beta}(m)\right|^{2}\right]$,

subject to $\sum_{n=1}^{N}\left|\hat{X}_{\alpha}(n)\right|^{2}=\sum_{n=1}^{N}\left|\hat{x}_{\beta}(n)\right|^{2}=1$.

To solve this problem let us consider the following Lagrangian

$$
\begin{aligned}
& L=-\sum_{n=1}^{N}\left[\left(\ln \left|\hat{x}_{\alpha}(n)\right|^{2}\right) \cdot\left|\hat{x}_{\alpha}(n)\right|^{2}\right]-\sum_{m=1}^{N}\left[\left(\ln \left|\hat{x}_{\beta}(m)\right|^{2}\right) \cdot\left|\hat{x}_{\beta}(m)\right|^{2}\right] \\
& +\lambda_{1}\left(\sum_{n=1}^{N}\left|\hat{x}_{\alpha}(n)\right|^{2}-1\right)+\lambda_{2}\left(\sum_{n=1}^{N}\left|\hat{x}_{\beta}(n)\right|^{2}-1\right) .
\end{aligned}
$$

In order to simplify the computation, we set $\left|\hat{x}_{\alpha}(n)\right|^{2}=p_{n}^{\alpha}$ and $\left|\hat{x}_{\beta}(n)\right|^{2}=p_{n}^{\beta}$. Hence we have

$$
\begin{aligned}
& \frac{\partial L}{\partial p_{n}^{\alpha}}=-\ln p_{n}^{\alpha}-1+\lambda_{1}=0, \\
& \frac{\partial L}{\partial p_{n}^{\beta}}=-\ln p_{n}^{\beta}-1+\lambda_{2}=0, \\
& \sum_{n=1}^{N} p_{n}^{\alpha}=1, \\
& \sum_{n=1}^{N} p_{n}^{\beta}=1 .
\end{aligned}
$$

Solving the above equations, we finally obtain $\left|\hat{x}_{\alpha}(n)\right|=\frac{1}{\sqrt{N_{\alpha}}},\left|\hat{x}_{\beta}(n)\right|=\frac{1}{\sqrt{N_{\beta}}}$. From the definition of Shannon entropy, we know that if $H\left(\hat{x}_{\alpha}\right)=\ln N_{\alpha}$ and $H\left(\hat{x}_{\beta}\right)=\ln N_{\beta}$, the equality in (3) holds. In addition, we also have $N_{\alpha} N_{\beta}=|N \cdot \sin (\alpha-\beta)|$. From the proof we know that $\left|\hat{x}_{\alpha}(n)\right|=\frac{1}{\sqrt{N_{\alpha}}}$ and $\left|\hat{x}_{\beta}(n)\right|=\frac{1}{\sqrt{N_{\beta}}}$ means that $\hat{x}_{\alpha}(m)$ and $\hat{x}_{\beta}(n)$ can be complex, and only if their amplitudes are constants, the equality will hold. Now we can obtain the following corollary out of above analysis.

Corollary 1: For any given discrete time series $\tilde{X}=\left\{x_{1}, x_{2}, x_{3}, \cdots, x_{N}\right\} \equiv\{x(1), x(2), x(3), \cdots, x(N)\} \in C^{N}$ with cardinality $N$ and $\|\tilde{X}\|_{2}=1, \hat{x}_{\alpha}\left(\hat{x}_{\beta}\right)$ is the DFRFT series associated with the transform parameter $\alpha$ ( $\beta$, respectively), $N_{\alpha}\left(N_{\beta}\right)$ counts the non-zero elements of $\hat{x}_{\alpha}\left(\hat{x}_{\beta}\right.$, respectively), if $\left|\hat{x}_{\alpha}(n)\right|=\frac{1}{\sqrt{N_{\alpha}}}$ and $\left|\hat{x}_{\beta}(n)\right|=\frac{1}{\sqrt{N_{\beta}}}$, then we have

$$
N_{\alpha} \cdot N_{\beta}=N \cdot|\sin (\alpha-\beta)|
$$

and

$$
H\left(\hat{x}_{\alpha}\right)+H\left(\hat{x}_{\beta}\right)=\ln (\mid N \cdot \sin (\alpha-\beta)) .
$$

\subsection{Rényi Entropic Principle}

Theorem 2: For any given discrete time series $X=\left\{x_{1}, x_{2}, x_{3}, \cdots, x_{N}\right\}=\{x(1), x(2), x(3), \cdots, x(N)\} \in C^{N}$ with cardinality $N$ and $\|X\|_{2}=1, \hat{x}_{\alpha}\left(\hat{x}_{\beta}\right)$ is the DFRFT series associated with the transform parameter $\alpha$ ( $\beta$, respectively), $N_{\alpha}\left(N_{\beta}\right)$ counts the non-zero elements of $\hat{x}_{\alpha}\left(\hat{x}_{\beta}\right.$, respectively), then we can obtain the generalized discrete Renyi entropic uncertainty relation

$$
H_{\vartheta}\left(\hat{x}_{\alpha}\right)+H_{\zeta}\left(\hat{x}_{\beta}\right) \geq \ln (|N \cdot \sin (\alpha-\beta)|)
$$

$$
\text { with } \frac{1}{2}<\zeta \leq 1 \text { and } \frac{1}{\zeta}+\frac{1}{\vartheta}=2 \text {. }
$$

where 


$$
\begin{aligned}
& H_{\vartheta}\left(\hat{x}_{\alpha}\right)=\frac{1}{1-\vartheta} \cdot \ln \left(\sum_{m=1}^{N}\left|\hat{x}_{\alpha}(m)\right|^{2 \vartheta}\right), \\
& H_{\zeta}\left(\hat{x}_{\beta}\right)=\frac{1}{1-\zeta} \cdot \ln \left(\sum_{n=1}^{N}\left|\hat{x}_{\beta}(n)\right|^{2 \zeta}\right),
\end{aligned}
$$

which are Rényi entropies.

Proof: In lemma 1 , set $q=2 \vartheta$ and $p=2 \zeta$, we have $\frac{1}{2}<\zeta \leq 1$ and $\frac{1}{\zeta}+\frac{1}{\vartheta}=2$. Then from lemma 1 , we obtain

$$
\left(\sum_{m=1}^{N}\left|\hat{x}_{\alpha}(m)\right|^{2 \vartheta}\right)^{\frac{1}{2 \vartheta}} \leq(N \cdot|\sin (\alpha-\beta)|)^{\frac{\zeta-1}{2 \zeta}} \cdot\left(\sum_{n=1}^{N}\left|\hat{x}_{\beta}(n)\right|^{2 \zeta}\right)^{\frac{1}{2 \zeta}} \text {. }
$$

Take the square of the above inequality, we have

$$
\left(\sum_{m=1}^{N}\left|\hat{x}_{\alpha}(m)\right|^{2 \vartheta}\right)^{\frac{1}{g}} \leq(N \cdot|\sin (\alpha-\beta)|)^{\frac{\zeta-1}{\zeta}} \cdot\left(\sum_{n=1}^{N}\left|\hat{x}_{\beta}(n)\right|^{2 \zeta}\right)^{\frac{1}{\zeta}} \text {. }
$$

Take the power $\frac{\zeta}{1-\zeta}$ of both sides in above inequality, we obtain

$$
\begin{aligned}
& \left(\sum_{m=1}^{N}\left|\hat{x}_{\alpha}(m)\right|^{2 \vartheta}\right)^{\frac{1}{\vartheta-1}} \leq(N \cdot|\sin (\alpha-\beta)|)^{-1} \cdot\left(\sum_{n=1}^{N}\left|\hat{x}_{\beta}(n)\right|^{2 \zeta}\right)^{\frac{1}{1-\zeta}}, \\
& \text { i.e., } \frac{(N \cdot|\sin (\alpha-\beta)|)^{-1} \cdot\left(\sum_{n=1}^{N}\left|\hat{x}_{\beta}(n)\right|^{2 \zeta}\right)^{\frac{1}{1-\zeta}}}{\left(\sum_{m=1}^{N}\left|\hat{x}_{\alpha}(m)\right|^{2 \vartheta}\right)^{\frac{1}{9-1}}} \geq 1
\end{aligned}
$$

Take natural logarithm in both sides of (5), we can obtain

$$
\begin{aligned}
& \frac{1}{1-\zeta} \cdot \ln \left(\sum_{n=1}^{N}\left|\hat{x}_{\beta}(n)\right|^{2 \zeta}\right)+\frac{1}{1-\vartheta} \cdot \ln \left(\sum_{m=1}^{N}\left|\hat{x}_{\alpha}(m)\right|^{2 \vartheta}\right) \\
& \geq \ln (|N \cdot \sin (\alpha-\beta)|) .
\end{aligned}
$$

Clearly, as $\zeta \rightarrow 1$ and $\vartheta \rightarrow 1$, the Renyi entropy reduces to Shannon entropy, thus the Renyi entropic uncertainty relation in (4) reduces to the Shannon entropic uncertainty relation (3). Hence the proof of equality in theorem 2 is trivial according to the proof of theorem 1 .

Note that although Shannon entropic uncertainty relation can be obtained by Rényi entropic uncertainty relation, we still discuss them separately in the sake of integrality.

\section{Resolution Analysis in Time-Frequency Domain}

In many cases [13-15], we often discuss the data concentration in both time domain and frequency domain, therefore, we will adopt a new measure on entropy:

$$
H_{p}=p H\left(\hat{x}_{\alpha}\right)+(1-p) H\left(\hat{x}_{\beta}\right),
$$

where $\hat{x}_{\alpha}\left(\hat{x}_{\beta}\right)$ is the DFRFT series associated with the transform parameter $\alpha$ ( $\beta$, respectively) for time series $\tilde{X}=\left\{x_{1}, x_{2}, x_{3}, \cdots, x_{N}\right\}=\{x(1), x(2), x(3), \cdots, x(N)\}, H\left(\hat{x}_{\alpha}\right)$ and $H\left(\hat{x}_{\beta}\right)$ are the Shannon entropies for the DFRFT series $\hat{x}_{\alpha}\left(\hat{x}_{\beta}\right)$.

Theorem 3: For any given discrete time series
$\tilde{X}=\left\{x_{1}, x_{2}, x_{3}, \cdots, x_{N}\right\}_{\tilde{N}}=\{x(1), x(2), x(3), \cdots, x(N)\} \quad$ with cardinality $N$ and $X \|_{2}=1, \hat{x}_{\alpha}\left(\hat{x}_{\beta}\right)$ is the DFRFT series associated with the transform parameter $\alpha$ ( $\beta$, respectively), $N_{\alpha}\left(N_{\beta}\right)$ counts the non-zero elements of $\hat{x}_{\alpha}\left(\hat{x}_{\beta}\right.$, respectively), then we can obtain the generalized discrete entropic uncertainty relation

$$
H_{1 / 2} \geq \ln (\sqrt{|N \cdot \sin (\alpha-\beta)|})
$$

Proof: For variable $\hat{x}_{\alpha}$, we have for $0<p<\infty$

$$
\left\|\hat{x}_{\alpha}\right\|_{p}=\left(\sum_{n=1}^{N}\left|\hat{x}_{\alpha}(n)\right|^{p}\right)^{\frac{1}{p}} \text {. }
$$

Set $\left\|\hat{x}_{\alpha}\right\|_{\infty}=\max _{n \in N}\left\{\left|\hat{x}_{\alpha}(n)\right|\right\}$. Therefore, we have

$$
\begin{gathered}
\frac{d}{d q} \log \left\|\hat{x}_{\alpha}\right\|_{\frac{1}{q}}=-\sum_{n=1}^{N} \frac{\left|\hat{x}_{\alpha}(n)\right|^{1 / q}}{\left\|\hat{x}_{\alpha}\right\|_{\frac{1}{q}}^{1 / q}} \log \left(\frac{\left|\hat{x}_{\alpha}(n)\right|^{1 / q}}{\left\|\hat{x}_{\alpha}\right\|_{1 / q}^{1 / q}}\right), \\
\frac{d^{2}}{d q^{2}} \log \left\|\hat{x}_{\alpha}\right\|_{\frac{1}{q}}^{1 / q} \\
\left.=\frac{1}{t} \sum_{n=1}^{N} \frac{\left|\hat{x}_{\alpha}(n)\right|^{1 / q}}{\left\|\hat{x}_{\alpha}\right\|_{1 / q}^{1 / q}} \log \left(\frac{\left|\hat{x}_{\alpha}(n)\right|^{1 / q}}{\left\|\hat{x}_{\alpha}\right\|_{1 / q}^{1 / q}}\right)-\sum_{n=1}^{N} \frac{\left|\hat{x}_{\alpha}(n)\right|^{1 / q}}{\left\|\hat{x}_{\alpha}\right\|_{1 / q}^{1 / q}} \log \left(\frac{\left|\hat{x}_{\alpha}(n)\right|^{1 / q}}{\left\|\hat{x}_{\alpha}\right\|_{1 / q}^{1 / q}}\right)\right)^{2} .
\end{gathered}
$$

Taking into account of the non-negative second derivative, therefore we know that $\log \left\|\hat{x}_{\alpha}\right\|_{\frac{1}{q}}$ is convex.
Therefore, we obtain

$$
\begin{aligned}
H\left(\hat{x}_{\alpha}\right) & =\left.\frac{d}{d q} \log \left\|\hat{x}_{\alpha}\right\|_{1 / q}\right|_{q=\frac{1}{2}} \leq \lim _{q \rightarrow+\infty} \frac{d}{d q} \log \left\|\hat{x}_{\alpha}\right\|_{1 / q} \\
& =\log (|N \cdot \sin (\alpha-\beta)|) .
\end{aligned}
$$

Since $\left\|\hat{x}_{\alpha}\right\|_{2}=\left\|\hat{x}_{\beta}\right\|_{2}=\|\tilde{X}\|_{2}=1$ and $\left\|\hat{x}_{\alpha}\right\|_{\infty} \leq \frac{1}{\sqrt{|N \cdot \sin (\alpha-\beta)|}}\left\|\hat{x}_{\alpha}\right\|_{1}$ and the Riesz-Thorin theorem [4], one has

$$
\frac{\left\|\hat{x}_{\alpha}\right\|_{\frac{1}{1-q}}}{\left\|\hat{x}_{\beta}\right\|_{\frac{1}{q}}} \leq|N \sin (\alpha-\beta)|^{\frac{1}{2}-q}\left(\frac{1}{2} \leq q \leq 1, \hat{x}_{\alpha} \neq 0\right)
$$

By applying negative logarithm to both sides of above inequality, we obtain the following relation

$$
\begin{aligned}
& \log \left(\left\|\hat{x}_{\beta}\right\|_{\frac{1}{q}}\right)-\log \left(\left\|\hat{x}_{\alpha}\right\|_{\frac{1}{1-q}}\right) \\
& \geq\left(q-\frac{1}{2}\right) \log (|N \sin (\alpha-\beta)|), \quad\left(\frac{1}{2} \leq q \leq 1\right) .
\end{aligned}
$$

Taking into account of $\left\|\hat{x}_{\alpha}\right\|_{2}=\left\|\hat{x}_{\beta}\right\|_{2}=\|\tilde{X}\|_{2}=1$, then both sides of above inequality are equal to zero for $q=\frac{1}{2}$. Hence, (7) and (8) imply

$$
\frac{1}{2} H\left(\hat{x}_{\alpha}\right)+\frac{1}{2} H\left(\hat{x}_{\beta}\right) \geq \frac{1}{2} \ln (|N \cdot \sin (\alpha-\beta)|) .
$$

Thus the proof is completed. Note that this proof can 
be obtained via the similar manner with Section 3 A, however, we still give a new proof so that we can understand this point out of different aspect. On the other hand, different proofs yield the same result, as validates the conclusion derived here.

\section{Conclusions}

In this article, we extended the entropic uncertainty relations in DFRFT domains. We first introduced the generalized discrete Hausdorff-Young inequality. Based on this inequality, we derived the discrete Shannon entropic uncertainty relation and discrete Rényi entropic uncertainty relation. Interestingly, when the variable's amplitude is equal to the constant, i.e. the inverse of the square root of number of non-zero elements, the equality holds in the uncertainty relation. In addition, the product of the two numbers of non-zero elements is equal to $N \cdot|\sin (\alpha-\beta)|$, i.e., $N_{\alpha} N_{\beta}=N \cdot|\sin (\alpha-\beta)|$.

\section{REFERENCES}

[1] R. Ishii and K. Furukawa, "The Uncertainty Principle in Discrete Signals,” IEEE Trans Circuits and Systems, Vol. 33, No. 10, 1986, pp. 1032-1034.

[2] L. C. Calvez and P. Vilbe, "On the Uncertainty Principle in Discrete Signals," IEEE Trans Circuits and Systems-II: Analog and Digital Signal Processing, Vol. 39, No. 6, 1992, pp. 394-395. doi:10.1109/82.145299

[3] S. Shinde and M. G. Vikram, “An Uncertainty Principle for Real Signals in the Fractional Fourier Transform Domain,” IEEE Transactions Signal Processing, Vol. 49, No. 11, 2001, pp. 2545-2548. doi:10.1109/78.960402

[4] G. L. Xu, X. T. Wang and X. G. Xu, "Generalized Entropic Uncertainty Principle on Fractional Fourier transform," Signal Processing, Vol. 89, No. 12, 2009, pp. 2692-2697. doi:10.1016/j.sigpro.2009.05.014

[5] R. Tao, B. Deng and Y. Wang, "Theory and Application of the Fractional Fourier Transform,” Tsinghua Univer- sity Press, Beijing, 2009

[6] S.C. Pei and J. J. Ding, "Eigenfunctions of Fourier and Fractional Fourier Transforms With Complex Offsets and Parameters,” IEEE Trans Circuits and Systems-I: Regular Papers, 2007, Vol. 54, No. 7, pp. 1599-1611.

[7] T. M. Cover and J. A. Thomas, "Elements of Information Theory, Second Edition,” John Wiley \&Sons, Inc.,2006.

[8] H. Maassen, “A Discrete Entropic Uncertainty Relation,” Quantum Probability and Applications, V, Springer-Verlag, New York, 1988, pp. 263-266.

[9] C. E. Shannon. "A Mathematical Theory of Communication,” The Bell System Technical Journal, Vol. 27, 1948, pp. 379-656

[10] A. Rényi, On Measures of Information and Entropy, In: Proceedings of the Fourth Berkeley Symposium on Mathematics, Statistics and Probability, 1960, p. 547.

[11] G. Hardy, J. E. Littlewood and G. Pólya Inequalities. 2nd edition, Press of University of Cambridge, 1951.

[12] D. Amir, T. M. Cover and J. A. Thomas, "Information Theoretic Inequalities,” IEEE Trans Information Theory, Vol. 37, No. 6, 2001, pp. 1501-1508.

[13] D. Victor, O. Murad and P. Tomasz, "Resolution in Time-Frequency," IEEE Transactions on Signal Processing, Vol. 47, No. 3, 1999, pp. 783-788. doi:10.1109/78.747783

[14] T. Przebinda, V. DeBrunner and M. Özaydın, “The Optimal Transform for the Discrete Hirschman Uncertainty Principle,” IEEE Transactions on information theory, Vol. 47, No. 5, 2001, pp. 2086-2090. doi:10.1109/18.930948

[15] X. D. Zhang, “Modern Signal Processing, “second edition) Tsinghua university press, Beingjing, 2002.

[16] G. L. Xu, X. T. Wang and X. G. Xu, "The Logarithmic, Heisenberg's and Short-time Uncertainty Principles Associated with Fractional Fourier Transform," Signal Processing, Vol. 89, No. 3, 2009, pp. 339-343. doi:10.1016/j.sigpro.2008.09.002 